\title{
Oral Diuretic Therapy Augmentation for Heart Failure
}

National Cancer Institute

\section{Source}

National Cancer Institute. Oral Diuretic Therapy Augmentation for Heart Failure. NCI Thesaurus. Code C119199.

Initiation or intensification of oral diuretics to treat heart failure. 